\title{
The Economy, Climate Change and Infectious Diseases: Links and Policy Implications
}

\author{
William Brock ${ }^{1,2} \cdot$ Anastasios Xepapadeas 3,4
}

Accepted: 3 July 2020 / Published online: 10 July 2020

(c) Springer Nature B.V. 2020

\begin{abstract}
This short paper provides a modeling framework for unifying the economy, climate change and the outbreak of infectious diseases such as the recent COVID-19 pandemic. We stress that continuous growth of consumption activities, capital accumulation and climate change could increase the potential of the epidemic, its contact number or the probability of its arrival. This framework of analysis allows us to think of infectious disease policies in two stages. In the short run, containment policies like social distancing could help to stop the epidemic. In the medium and the long run, economic policies could help to reduce the potential of the epidemic or the probability of its emergence.
\end{abstract}

Keywords SIR model $\cdot$ Epidemic $\cdot$ Climate change $\cdot$ Containment policy $\cdot$ Regime shift

\section{Introduction}

The COVID-19 pandemic has generated a very large volume of research related to its epidemiology, vaccination and economic impacts in just a short period of time. ${ }^{1}$ In terms of economics, most of the research is focused on macroeconomic impacts and costs of the pandemic and its containment.

In terms of the environment, it seems that the containment policies led to a sharp reduction in $\mathrm{CO}_{2}$ emissions and other air pollutants. According to a very recent International Energy Agency (2020) report, global $\mathrm{CO}_{2}$ emissions are expected to decline to $30.6 \mathrm{GtCO} 2$ during 2020, which is $8.3 \%$ lower than in 2019 (36.8 GtCO2) (Global

\footnotetext{
${ }^{1}$ See, for example, the websites at https://www.nature.com/collections/hajgidghjb and https://www.scien cemag.org/collections/coronavirus? IntCmp=coronavirussiderail-128. An interesting collection of COVID19 economics papers can be found at: https://bfi.uchicago.edu/insight/blog/key-economic-facts-about-covid -19/\#targeted-lockdowns.
}

Anastasios Xepapadeas

xepapad@aueb.gr

1 University of Wisconsin, Madison, WI, USA

2 University of Missouri, Columbia, MO, USA

3 University of Economics and Business, Athens, Greece

4 University of Bologna, Bologna, Italy 
Carbon Project 2019). This would be the lowest level since 2010, and six times larger than the previous record reduction of $0.4 \mathrm{Gt}$ in 2009 due to the financial crisis. Coal use fell by $40 \%$ at China's six largest power plants since the last quarter of 2019, while in Europe satellite images show nitrogen dioxide $\left(\mathrm{NO}_{2}\right)$ emissions fading away over northern Italy, with a similar picture in Spain and the UK (Henriques 2020).

The issue of whether these reductions will persist over time or whether the opening of the economies after the lockdown will return emissions to their pre-pandemic paths is an open and interesting research issue. In this paper, however, we focus on a different issue, which evolves in a longer time horizon than the reduction of emissions after a short-run containment policy. This is the link between Emerging Infectious Diseases (EIDs) on the one hand, and climate change and the increasing human encroachment in the natural environment resulting in pressures on disease reservoirs and continued antibiotic resistance on the other. Human encroachment on wildlife with resulting stresses causing increased EIDs is emphasized, for example, by Daily and Ehrlich (1996) and Muehlenbein (2013). The main argument is that increased use of wildlife products, increasing use of concentrated animal husbandry, increasing human encroachment on nature, and land use changes cause the emergence of a small cluster of individuals infected by a novel disease, e.g., COVID-19 for the most recent example. A recent article (Harvey 2020) quotes primatologist Jane Goodall as saying that "Our disrespect for wild animals and our disrespect for farmed animals has created this situation where disease can spill over to infect human beings." She points to habitat destruction, the farming and consumption of wild animals, illegal wildlife trafficking and factory farming as risky practices that could fuel future pandemics. In the same spirit, in a Science Alert article (Ramon 2020), ecologist David Lapola warns that the next pandemic could come from the Amazon rainforest since human encroachment on animals' habitats-a likely culprit in the coronavirus outbreak-is soaring there because of rampant deforestation. In a similar vein, Afelt et al. (2018) — even before the current COVID-19 crisis-linked deforestation with the emergence of coronaviruses and novel infectious diseases, and Zimmer (2019) indicated that scientific evidence suggests that deforestation is leading to more infectious diseases in humans.

The purpose therefore of this short paper is to provide a conceptual framework for linking the human activities resulting in encroachment of natural habitats and climate change with EIDs and the associated containment policies. Our purpose is not to provide a detailed integrated assessment model with calibrations and a number of specific policy solutions, but rather to provide modeling ideas and approaches which could be used to develop more complex and detailed modeling.

We discuss a model of a one-time regime change where the world is disease free in the first regime but, because of pressures on disease reservoirs and continued antibiotic resistance associated with human activities, at a random time $\tau$ a COVID-19-like disease emerges with an initial cluster of infectives of size $\varepsilon$.

Our model unifies the consumption, capital accumulation, energy and climate components characterizing coupled models of the economy and the climate with a Susceptible, Infected, Recovered (SIR) model of an emerging infectious disease at a random time. The SIR component is added to capture the potential impact of anthropogenic "forcing" resulting in stresses causing increased EIDs and the need for costly containment. As we have seen from the devastating impacts of COVID-19 on the economy, it could be worth exploring possible channels between consumption and capital accumulation activities, along with climate change, on sources of EIDs. 


\section{Unifying the Environment, Climate Change and the Emergence of Infectious Diseases}

We conduct an initial modeling exercise in which a social planner seeks to maximize discounted utility subject to the economy, climate and SIR dynamics and the possibility of an infectious disease emerging at a random time $\tau$. In order to make the modeling approach clear, we start by assuming the infectious disease appears at the beginning of the planning horizon.

Following Barnett et al. (2020a), we consider an instantaneous utility function which is the logarithm of a Cobb Douglas specification of a material consumption aggregate $C$ and energy $E$. Utility is damaged by a positive temperature anomaly $T$ relative to the preindustrial period $T_{0}$ and by the infected part of the population $I$. Thus

$$
u=a \ln E+(1-a)\left(\ln C-a_{T} T-a_{I} I\right),
$$

where $a_{T} T, a_{I} I$ reflect damages to the consumption aggregate from climate change and the epidemic respectively, and $0<a<1$ is a preference parameter that determines the relative importance of energy in the instantaneous utility function.

The foundation of such a utility function can be traced back to Becker (1965). More specifically, following Becker (1965) we assume that utility is a function $\ln [f(E, M, H, Q)]$ where $\ln (f)$ is the natural $\log$ arithm of $f, E$ is energy, $M$ is market goods, $H$ is health, and $Q$ is environmental quality. We assume that $H=H_{0} \exp \left(-a_{I} I\right), Q=Q_{0} \exp \left(-a_{T} T\right]$ where $H_{0}, Q_{0}$ are initial states of health and environment and the $\exp (\cdot)$ terms are damages to health caused by infections and increases in temperature above the preindustrial temperature. If we put $M=C$ for market goods and assume a Cobb Douglas specification for the $E$ and $C \mathrm{e}^{-a_{I} I} \mathrm{e}^{-a_{T} T}$ components, then the utility function for the planner's preferences will be given by (1). ${ }^{2}$

Thus a planner's problem can be written as:

$$
\begin{gathered}
\max _{E, C} \int_{0}^{\infty} \mathrm{e}^{-\rho t}\left[a \ln E+(1-a)\left(\ln C-a_{T} T-a_{I} I\right)\right] d t \\
\text { subject to } \\
C+\dot{K}+\delta K=A K, K(0)=K_{0} \\
\dot{R}=-E, R(0)=R_{0} \\
\dot{T}=b E-m T, T(0)=T_{0} \\
\dot{S}=-\beta(C, T) \frac{S I}{M}, S(0)=S_{0} \geq 0 \\
\dot{I}=\beta(C, T) \frac{S I}{M}-\gamma I, I(0)=I_{0} \geq 0,
\end{gathered}
$$

\footnotetext{
${ }^{2}$ This specification is the same as the specification in Barnett et al. (2020a) when health is included.
} 
where $K$ is the stock of capital in the economy for which an $A K$ production function is assumed; $R$ is the known reserve of fossil fuels from which energy $E$ (or emissions) is "extracted"; $T$ is the temperature anomaly, with $m$ a dissipation parameter. Note also that $S$ is susceptible, $I$ is infected, $M$ is population, $\gamma$ is the recovery rate and $\beta$ is the contact number. The temperature anomaly dynamics follows an approximation as in Matthews et al. (2009) who show that the anomaly generated by mainstream Atmosphere Ocean General Circulation Models approximately scales linearly with cumulative carbon emissions into the atmosphere. Cline (2020, Appendix C and main text) argues that the approximate linearity of the global temperature anomaly response is approximately linear in cumulative emissions because of three effects: "the legacy effect, the atmospheric retention effect, and the rising emissions effects." The legacy effect refers to the behavior of the log function in added atmospheric carbon beyond the initial stock of atmospheric carbon, the atmospheric retention effect refers to the weakening of the carbon sinks as more carbon is added, ${ }^{3}$ and the rising emissions effect refers to the convexity added when an emissions path is integrated from date zero to date $t$. Brock and Xepapadeas (2017) provide an early application of linear anomaly dynamics to a coupled model of climate and economy.

Equation (4) suggests a proportionality relationship between the energy component and emissions with units chosen so that the proportionality factor is one. Data sources indicate that emissions are not strictly proportional to energy but they are highly correlated. From Greenstone et al. (2019, Figure 1), for example, we see that log GDP and log energy are highly correlated. Hence, if emissions and output are highly correlated, then it is plausible that emissions and energy are highly correlated. Evidence in support of the positive correlation between energy and emissions is provided by data from the World Bank (2020). Although in the long run substitution away from fossil fuels and their emissions towards cleaner sources of energy will occur under the right incentives, it can be argued that as a "first cut," a Leontief assumption for emissions and energy plus a Leontief assumption for renewables (which have zero emissions, including nuclear and biofuels as renewables) could roughly approximate reality on a time scale before major substitution away from fossil-fuel-emitting energy sources takes place. World Bank data suggest that although substitution is projected to take place, fossil fuel emissions are projected to continue to be large for a long time. 4

The material consumption aggregate, which is affected by climate change and the EIDs, is regarded as a function of services yielded by not only consumption of household goods and services but also travel, cruising, ecotourism, land use change and so on, which puts humans into increasing contact with wildlife and which pressures wildlife already stressed by increasing human encroachment. This means that part of the consumption bundle increases the disease channels stressed by Daily and Ehrlich (1996) and Muehlenbein (2013).

In more detail, the consumption aggregate $C$ can be constructed by using an approach like the Becker/Lancaster activity analysis approach to building utility functions, e.g., as in Pollak and Wachter (1975). Becker and Lancaster put utility on a vector of activities produced via a neoclassical production function for each activity that used time and a vector of

\footnotetext{
3 See also MacDougall and Friedlingstein (2015, Figures 4-6) for climate scientific support of this effect.

${ }^{4}$ In an initial simple prototype model as stark as this one, we can expect only to capture rough features of reality. A richer model would include resources spent to increase the elasticity of substitution between dirty energy sources (e.g., coal, oil and gas) and clean energy sources (e.g., wind, solar, and maybe nuclear if the waste disposal problem could be solved, and maybe hydro if damages could be reduced).
} 
material goods. This approach is much richer and allows one to build aggregates in interesting ways that can be inserted as a component into the utility function in objective (2).

Thus, using the Becker/Lancaster approach to demand theory, together with fixed proportions of a vector of activities-e.g., ecotourism, land use changes, building second homes near wildlife habitats, burning down swaths of the Amazon for cattle ranches and soybean farms, ruining swaths of jungle for palm oil plantations - a scalar aggregate which is called " $C$ " here can be built. This aggregate is damaged by climate change and the epidemic, but at the same time contributes to the epidemic by assuming that the pressures on sources of EIDs emphasized by researchers such as Daily and Ehrlich (1996) and Muehlenbein (2013) roughly scale with aggregate consumption $C$.

While we know qualitatively that increasing encroachment by humans on wildlife habitats and increased consumption of wildlife products, as well as increased concentration and scale of animal husbandry (e.g., Confinement Animal Feeding Operations or CAFOs), are associated with increased EIDs, this is not enough. We need to be able to address the amount of increased EIDs associated with such activities.

In this context it makes sense to talk about increasing aggregate world consumption increasing the rate of emergence of EIDs. Also important is the scaling up of industrial agriculture and factory farming which has lowered antibiotic resistance because of antibiotic usage in CAFOs. This lowering of effectiveness in the disease-fighting arsenal could be treated as increasing the epidemic contact rate and the fundamental parameter $R_{0}$ of an epidemic.

This link between aggregate world consumption and rate of emergence of EIDs can be modeled using Eqs. (6) and (7). ${ }^{5}$ These equations represent the SIR component of the unified model. Recall that $S$ is susceptible, $I$ is infected, $M$ is population, $\gamma$ is the recovery rate and $\sigma(C, T)=\beta(C, T) / \gamma$ is the contact number defined as the contact rate $\beta(C, T)$ divided by the average infectious period $1 / \gamma$. The lowering of effectiveness in the disease-fighting arsenal could be treated as increasing $\beta$ which we assume to be an increasing function of $C$ and $T$. This is used to capture the increasing encroachment on wildlife habitats, increasing stress and contacts on and with wildlife by humans (which might also weaken wildlife immune systems), as well as increased rates of spreading disease through traveling, crowding and other pressures related to the increasing scale of human consumption activities that are associated with the spreading of disease and increasing EIDs. Daily and Ehrlich (1996) and Muehlenbein (2013) suggest that $\beta$ should increase in $C$ and $T$, and maybe even have a positive cross partial derivative.

Assuming that all of the population is susceptible, the basic reproduction number $R_{0}$ which policy is trying to reduce below 1 is $\sigma(C, T)$. Then the regulation of an epidemic can be based on the following result (e.g., Hethcote 2000). Let $s=S / M$ and $i=I / M$ so that $s+i \leq 1$, and consider the system

$$
\dot{s}=-\beta i s, s(0)=s_{0} \geq 0
$$

\footnotetext{
5 We consider that our Eqs. (6) and (7) for the SIR model, which do not include the $d R(t) / d t$ equation, are a good enough approximation and that they simplify the analysis by saving the analytical cost of an extra state variable, since the size of the Earth's population suggests that the number of deaths from COVID19 is small relative to the global population. Therefore, ignoring the "feedback" of $d R(t) / d t$ to the $d S / d t$ equation can be regarded as a workable approximation. Furthermore $R=M-S-I$ and, dividing by $M$, $r=1-s-i$.
} 


$$
\begin{gathered}
\dot{i}=\beta i s-\gamma i, i(0)=i_{0} \geq 0 \\
Z=\{(s, i): s \geq 0, i \geq 0, s+i \leq 1\} .
\end{gathered}
$$

Also assume that $s_{o}=1$. Then by Hethcote's (2000) theorem 2.1, we have:

Let $(s(t), i(t))$ be a solution of (8)-(10) in $Z$. If $R_{0}=\sigma=\beta / \gamma \leq 1$ then $i(t)$ decreases to zero as $t \rightarrow \infty$. If $\sigma>1$, then $i(t)$ first increases up to a maximum value $i_{\text {max }}=i_{o}+1-(1 / \sigma)-[\ln (\sigma)] / \sigma$ and then decreases to zero as $t \rightarrow \infty$. The susceptible fraction $s(t)$ is a decreasing function and the limiting value $s_{\infty}$ is the unique root in $(0,1 / \sigma)$ of the equation

$$
i_{o}+1-s_{\infty}+\ln \left(\frac{s_{\infty}}{s_{o}}\right) / \sigma=0
$$

To reduce the damages from the epidemic, containment is introduced. The objective of the containment is to attain $\sigma<1$. Containment implies a control procedure which would reduce $\beta$. Thus we enrich the model by inserting a control " $v$ " and redefining $\sigma$ as $\sigma(v, C, T)$. The control is available at a convex increasing cost function $c(v)$. We define a "good" policy as follows.

Definition A "good" policy is a choice of $\left\{v_{i}, C_{t}, E_{t}\right\}_{t=0}^{\infty}$ such that $\sigma(v, C, T)$ is less than or equal to 1 for all $t \geq 0$.

We explore now whether the unified system (2)-(7) can be used to control the epidemic - in addition to controling consumption and energy use-in the spirit of Hethcte's (2000) theorem 2.1, that is, by reducing $\beta(v, C, T) / \gamma$ below 1 by a suitable choice of $v$.

\subsection{Simplified Good Policies: A Conceptual Framework}

We start by assuming that $\sigma(v, C, T)$ does not depend on $(C, T)$. In this case, $R_{0}$ under a containment policy $\phi(v)$ can be written to simplify notation as $R_{0}=\phi(v) \sigma=\phi(v) \beta / \gamma=\beta(v) / \gamma$, with $\beta^{\prime}(v)<0$, along with the requirement that $\beta(v) / \gamma \leq 1$. Containment policy has a known convex cost $c(v)$.

Normalizing the population to one in order to simplify, so that $s=S, i=I$, and using (8)-(9), we can obtain

$$
\frac{d I}{d S}=-1+\frac{\gamma}{\beta(v) S}
$$

with solution curves for the infected as a function of the susceptible which include the containment policy $v:^{6}$

$$
I(v, S)=1-S+\frac{\gamma}{\beta(v)} \ln \left(\frac{S}{S_{0}}\right)
$$

${ }^{6}$ See, for example, Hethcote (1976) without the containment component. 
The dynamics for the susceptible can then be written for $S(0)=1, I(0)=0$, as: $^{7}$

$$
\dot{S}=-\beta(v) S\left[1-S+\frac{\gamma \ln S}{\beta(v)}\right], S(0)=1, I(0)=0 .
$$

Then, $S(0)=1, I(0)=0$ is a steady state for (12) for each fixed $v$. Furthermore, this steady state is stable if $\frac{\partial}{\partial S}\left[-\beta(v) S\left(1-S+\frac{\gamma \ln S}{\beta(v)}\right)\right]_{S=1}<0$ which is true if and only if $R_{0}=\frac{\beta(v)}{\gamma}<1$. This provides a framework for analyzing good policies. We formulate some possibilities below.

1. Since the SIR component separates from the $A K, C, K$ part of problem (2), the following reduced problem based on (12) can be considered:

$$
\min _{v} \int_{0}^{\infty} e^{-\rho t}\left[(1-a) a_{I} I(v, S)+c(v)\right] d t
$$

subject to

$$
\begin{gathered}
\dot{S}=-\beta(v) S\left[1-S+\frac{\gamma \ln S}{\beta(v)}\right], S(0)=1, I(0)=0 \\
I(v, S)=1-S+\frac{\gamma \ln S}{\beta(v)} .
\end{gathered}
$$

2. Since $S(0)=1, I(0)=0$ is a steady state for (12) for each fixed $v$, the simpler static optimization problem

$$
\begin{gathered}
\min _{v}\left[(1-a) a_{I} I(v, S)+c(v)\right] \\
\text { subject to } \\
0 \leq \frac{\beta(v)}{\gamma} \leq 1
\end{gathered}
$$

can also be considered. Then it needs to be shown that for a candidate optimal solution $v^{*}$ from problem (16), this $v^{*}$ is also optimal for problem (13).

Solutions of the above problems, and in particular the issue of whether the solution of problem (16) is an optimal solution for problem (13), is left for further research. Further research can also explore cases in which the containment cost reduces output instead of affecting utility. In this case a link is generated between the SIR and the $A K$ model through the output budget constraint.

In the simple models described here, the link between the epidemic and the economy was realized through the damage of the epidemic on the consumption aggregate and the cost of the containment policy. The next step is to allow the economy and its influence through encroachment on nature and climate change to affect the contact rate $\beta$.

\footnotetext{
7 To be completely rigorous, initial conditions should be replaced with $S(0)=1-\varepsilon, I(0)=\varepsilon$, for small positive $\varepsilon$ and consider the case $\varepsilon \rightarrow 0$.
} 


\section{Consumption-Climate-Containment Policies}

To study the link between consumption, climate change and containment policies, we introduce the concept of the EID "potential" defined as

$$
\max \{0, f(C, T)-g(C, T) v\},
$$

where $f(C, T), g(C, T)$ are positive and increase (decrease) in $(C, T)$. Writing the containment policy as $\phi(v)=\max \{0, f(C, T)-g(C, T) v\}$ and assuming that the initial reserve $R_{0}$ is infinite, the corresponding Hamiltonian function for the problem is:

$$
\begin{aligned}
\mathcal{H}=[a & \left.\ln E+(1-a)\left(\ln C-a_{T} T-a_{I} I\right)-c(v)\right]+\mu_{K}(A K-C-\delta K) \\
& +\mu_{S}(-\phi(v) \beta(C, T) S I)+\mu_{I}[\phi(v) \beta(C, T) S I-\gamma I] \\
& +\mu_{T}(b E-m T)+\mu_{R}(-E) .
\end{aligned}
$$

It is interesting to notice that in this case both the consumption aggregate and climate are interacting with the epidemic. To obtain a clearer picture of potential policy schemes consistent with the optimization problem associated with the Hamiltonian function (18), assume that the containment costs are linear $c(v)=\omega v$ and there are upper bounds on the control $v$ of the form $0 \leq v \leq v_{\max }$. Then the Hamiltonian is linear in the epidemic control and we have a "bang-bang" type of solution for the containment policy, or

$$
\begin{gathered}
\text { If } g \beta S I\left(\mu_{S}-\mu_{I}\right)>\omega \Rightarrow v=v_{\max }, \dot{I}=I \gamma\left[\left(f-g v_{\max }\right) \frac{\beta}{\gamma} S-1\right] \\
\text { If } g \beta S I\left(\mu_{S}-\mu_{I}\right)<\omega \Rightarrow v=0, \dot{I}=I \gamma\left[f \frac{\beta}{\gamma} S-1\right] .
\end{gathered}
$$

Note that to ease notation we write $f, g, \beta$ instead of $f(C, T), g(C, T), \beta(C, T)$ respectively. We are interested in a steady state, $S=1, I=0$. To obtain this, the policy should be

$$
\begin{gathered}
\left(f-g v_{\max }\right) \frac{\beta}{\gamma} S<1 \text { when } v=v_{\max } \\
f \frac{\beta}{\gamma} S<1 \text { when } v=0 .
\end{gathered}
$$

Since the right hand side of the inequalities are functions of $(C, T)$, we can consider the contours of functions $h\left(C, T ; S,\left\{v_{\max }\right.\right.$ or 0$\left.\}\right)$ in the $(C, T)$ space parametrized by $S \in(0,1],\left\{v_{\max }\right.$ or 0$\}$. These contours will represent combinations of consumption policies in terms of the consumption aggregate, and climate policies in terms of the temperature anomaly, which will keep $R_{0}<1$. These consumption and climate policies will not be optimal in the sense of the full optimality conditions associated with (18), but they will support the optimal containment policy. Thus (21)-(22) can be regarded as a combined consumption-climate-containment policy which can stop the epidemic.

In general if $v$ is always chosen, so that $\phi(v)<0$-i.e., so that $S=1, I=0$ in a steady state-then a "quasi-optimal" policy is to choose a policy such that $\phi(v ; C, T)=0^{+}$where $0^{+}$denotes the limit from the right, which we think of as "barely positive." This policy keeps the EID from emerging, using control $v$ at a minimum cost. However, this policy 
may not be optimal for several reasons. First, the cost function $c(v)$ may increase rapidly as $v$ increases, especially if there is a capacity constraint. Second, the variables $(C, T)$ may be viewed as longer-time scale variables, but the anticipation of a forward-looking planner is defined in terms of shorter time scales, especially regarding the potential damages of the EID if containment fails.

The current COVID-19 epidemic, which has a large effect on $A$ in the $A K$ model, as well as a very large effect on $C$, may cause the planner to operate both $v$ and $C$-and potentially $T$ - simultaneously. This is the case of the "bang-bang" policy described by (19)-(20) combined with (21)-(22). The fully optimal policy on $(v, C, T)$ requires the solution of a more complex optimization problem characterized by optimality conditions stemming from the maximum principle corresponding to $(18){ }^{8}$

\section{Random Arrival of an Epidemic}

Our analysis up to this point was based on the underlying assumption that the epidemic has already started, so the issue is how to control an existing epidemic, such as COVID19. If, however, we consider the problem looking forward, an important issue is whether increased human expansion and increased anthropogenic pressures on the natural environment increase the probability of emergence of an epidemic. We model this by changing the structure above and analyzing the control of the epidemic as a regime change problem, much like Polasky et al. (2010) and van der Ploeg and de Zeeuw (2019) who assume that their hazard function depends on the state of the system before the regime change occurs and, once the regime change occurs, there are no further regime changes.

\subsection{The Cost of the Arrival}

First we study the impact of a small cluster of infected individuals after the arrival of the disease. The main difference from the previous modeling is that the SIR dynamics after the arrival of the disease are initialized at $I(\tau)=\varepsilon, S(\tau)=1-\varepsilon$, whereas previous to $\tau$, $I(\tau)=0, S(\tau)=1$. That is, no SIR dynamics were present.

We start by differentiating the post $\tau$ dynamics w.r.t. $\varepsilon$ evaluated at $\varepsilon=0$ to try to understand the marginal impact of a small cluster of infected individuals. Set $\tau=0$ and abstract from capital accumulation and consumption of $C$ to provide the simplest possible example. To simplify the problem, we abstract away from the problem of optimally accumulating capital inputs and we assume that capital accumulation and consumption paths evolve exogenously, in order to focus on costs from climate change and the infectious disease. Define

$$
\begin{aligned}
& J(\varepsilon) \\
& \quad=\int_{0}^{\infty} \mathrm{e}^{-\rho t}\left[\eta \ln E(t, \varepsilon)-(1-\eta)\left(a_{T}(T(t, \varepsilon))+a_{I}(I(t, \varepsilon))\right)\right] d t
\end{aligned}
$$

\footnotetext{
${ }^{8}$ Note that assuming that the initial reserve $R_{0}$ is infinite implies that $\mu_{R}=0$.
} 


$$
\begin{gathered}
\text { subject to } \\
\dot{T}(t, \varepsilon)=b E(t, \varepsilon)-m T(t, \varepsilon) \\
\dot{S}(t, \varepsilon)=-\phi(v) \beta S(t, \varepsilon) I(t, \varepsilon) \\
\dot{I}(t, \varepsilon)=\phi(v) \beta S(t, \varepsilon) I(t, \varepsilon)-\gamma I(t, \varepsilon) \\
I(0, \varepsilon)=\varepsilon, S(0, \varepsilon)=1-\varepsilon, \phi(v)=f-g v,
\end{gathered}
$$

where $a_{T}(T(t, \varepsilon)), a_{I}(I(t, \varepsilon))$ are climate change and epidemic costs respectively. The cost of the epidemic's arrival, which starts with a small cluster of infected individuals of size $\varepsilon$, can be approximated by the derivative $J^{\prime}(\varepsilon)$ along the optimal paths associated with the optimization problem (23)-(27) evaluated at $\varepsilon=0$.

Proposition 1 In the context of the simplified model (23)-(27) with linear climate change damages, the cost of the arrival of a pandemic which starts with a small cluster $\varepsilon$ of infected individuals is:

$$
\left.J^{\prime}(\varepsilon)\right|_{\varepsilon=0}=\frac{-(1-\eta)(d a(I) / d I)}{(\rho+\gamma-\beta f)} .
$$

For the proof, see the "Appendix".

The significance of this proposition is that it suggests that if the marginal damage parameter $d a(I) / d I$ of the epidemic is large, then the intrinsic contact rate $\beta$ is large, perhaps due to continued population growth (which causes increased congestion and encroachment on nature) and the past history of human activities. We see that the absolute value of marginal utility loss due to a small cluster of infectious individuals, could be extremely large if $\rho+\gamma-\beta f$ is close to zero. Indeed, the marginal utility loss could go to negative infinity, or not even be defined unless the subjective discount rate $\rho$ plus the recovery rate $\gamma$ exceeds the compound contact rate in $\beta f .{ }^{9}$ While the units in (28) are in utils, we may divide by the marginal utility of consumption at date zero to get the marginal damages in units of consumption.

Note that all actions of the control are missing, since the calculation in (28) is marginal around the no epidemic past. A component of epidemic control that has been shown by Greenstone and Nigum (2020) to be incredibly valuable is effective social distancing. They estimate the value of social distancing to be about $\$ 60,000$ per household in the U.S. Work such as that of Greenstone and Nigum (2020) suggests that if the structure of work could be reconfigured to implement effective social distancing but without provoking a large loss in total factor productivity, the gains to the economy of such innovation could be very large.

\subsection{Random Epidemic Arrivals: A Regime Shift Preliminary Approach}

In this section we introduce the idea of analyzing the arrival of an epidemic in the context of a regime shift approach, as done by Polasky et al. (2010) and van der Ploeg and

\footnotetext{
${ }^{9}$ We assume no deaths in the epidemic-despite the approximately $2 \%$ death rate from COVID-19-in order to avoid the controversial issues of attaching numbers to the value of human life.
} 
de Zeeuw (2019), using the concept of a hazard rate. In our approach, the hazard rate is endogenous and depends on the state of the economy and climate change. Given the complexity of the problem - and the objective of this special issue-we do not provide explicit solutions, but rather provide a setup which, given the importance of the problem and the large number of possible extensions, could provide the basis for areas of further research.

The basic idea is to consider two regimes: the one before $(\mathcal{B})$ and the one after $(\mathcal{A})$ the arrival of the epidemic. The arrival of the epidemic is determined by a hazard function $\lambda(K, T)$ which is assumed to depend upon climate change via the temperature anomaly and the capital stock. It seems more plausible to index the pressure of encroachment on wildlife habitats, deforestation, increased interaction of humans with wildlife EID reservoirs, increased concentration of animal husbandry, increased development of tourism and expanding infrastructure with capital stock rather than with consumption, since consumption could be dramatically decreased during an epidemic but the capital stock would still remain in the short run. Arguments related to deforestation as a strong driver of emerging highly pathogenic diseases (e.g., Afelt et al. 2018; Nabi et al. 2020) ${ }^{10}$ suggest that an index of the amount of deforestation could be developed which would be a good explanatory variable to put in the hazard function. Deforestation should be highly correlated with anthropization. Both of these should be strong drivers of emerging highly pathogenic diseases. This means that the determinants of the hazard function could include more drivers. ${ }^{11}$

There is a difference, however, between the climate regime shift approach of Polasky et al. (2010) and van der Ploeg and de Zeeuw (2019) and the epidemic associated regime shift. In the climate regime shift, the state variables of the problem are the same both before and after the regime shift. In contrast, in the analysis of the epidemic arrival, the state variables of the problem before the epidemic do not include the SIR model, while the SIR model is part of the setup of the problem after the epidemic. More specifically, in the context of the optimization problem associated with the Hamiltonian function (18), the before and after the epidemic value functions can be written as:

$$
\begin{aligned}
& \rho V^{\mathcal{B}}(K, T, S, I, R)=\max _{C, E}\left\{\left[a \ln E+(1-a)\left(\ln C-a_{T} T\right)\right]+V_{K}^{\mathcal{B}}(A K-C-\delta K)\right. \\
& \left.\mid V_{T}^{\mathcal{B}}(b E-m T)+V_{R}^{\mathcal{B}}(-E)-\lambda(K, T)\left[V^{\mathcal{B}}(K, T, S, I, R)-V^{\mathcal{A}}(K, T, S, I, R)\right]\right\} \\
& \rho V^{\mathcal{A}}(K, T, S, I, R)=\max _{C, E, v}\left\{\left[a \ln E+(1-a)\left(\ln C-a_{T} T-a_{I} I\right)-c(v)\right]\right. \\
& \quad+V_{K}^{\mathcal{A}}(A K-C-\delta K)+V_{T}^{\mathcal{A}}(b E-m T)+V_{R}^{\mathcal{A}}(-E)+V_{S}^{\mathcal{A}}(\phi(v) \beta(C, T) S I) \\
& \left.\quad+V_{I}^{\mathcal{A}}[(\phi(v) \beta(C, T) S I-\gamma I)]\right\} .
\end{aligned}
$$

The solution of the optimization problems associated with the two value functions above is beyond the scope of the present paper; the setup, however, suggests a number of different areas for further research depending on the complexities or simplifications which could be introduced and the calibration capabilities.

\footnotetext{
10 Nabi et al. (2020) suggest that to prevent bat-born viral outbreaks, policies should be focused on forestation, controlling population growth, bat hunting and consumption, a global ban on wildlife trade, surveillance and monitoring and ecological research.

11 An interesting area of future research could be fitting hazard functions to deforestation indices.
} 


\section{Concluding Remarks}

The purpose of this paper is to provide a modeling framework for unifying the economy, climate change and the outbreak of infectious diseases such as the recent COVID-19 pandemic. The basic idea is that the continuous growth of consumption activities, capital accumulation and greenhouse gas emissions, which result in climate change, could increase the potential of an epidemic, its contact number or the probability of its arrival. This framework of analysis allows us to think of infectious disease policies in two stages. In the short run, containment policies such as social distancing could help bring down $R_{0}$ and stop the epidemic. In the medium and the long run, economic policies aimed at changing consumption patterns and addressing climate change could help to reduce the potential of the epidemic or the probability of its emergence.

Clearly there are many ways of extending the basic model. One obvious and very realistic one is to allow for uncertainty. For example, worldwide estimates of $R_{0}$ provided by the website Worldometers ${ }^{12}$ for COVID-19 range from 1.4 to 4.0, while recent estimates of the same parameter for different areas of England range from 0.72 to 1.29 (Birrell et al. 2020). Moreover Shaw (2020) indicates that the basic reproduction number of SARS-CoV-2 (the virus that causes COVID-19), originally thought to be in the 2-3 range, may be closer to 5 or 6 . Furthermore, stochastic versions of the SIR models in which a Wiener process is attached to the deterministic SIR equations are common (e.g., Tornatore et al. 2005; Barnett et al. 2020b). It is clear that concepts related to the "trinity of uncertainty" (Brock and Hansen 2018) and robust control methods can be applied in such cases.

Acknowledgements We would like to thank an anonymous referee for valuable comments on an earlier draft of this paper. William Brock thanks RDCEP (Robust Decision Making on Climate and Energy Policy) at the University of Chicago under National Science Foundation Grants SES-0951576 and SES-1463644 for support of some of his work cited in this article Anastasios Xepapadeas thanks the AUEB Research Center Program 3049-01 for support of this work.

\section{Appendix}

Proof of Proposition 1 When evaluating at $\varepsilon=0, I=0$ and $S=1$, it follows that $v=0$ since there is no epidemic to control. At $\varepsilon=0$, the following derivatives along optimal paths can be calculated: calculated:

$$
\begin{gathered}
\frac{d \ln I^{\prime}(t, 0)}{d t}=\beta \phi(0)-\gamma \\
\frac{\eta E^{\prime}(t, 0)}{(E(t, 0))^{2}}=b \mu_{T}^{\prime}(t, 0) \\
\dot{\mu}_{T}=(\rho+m) \mu_{T}+(1-\eta) \frac{d a_{T}(T)}{d T}
\end{gathered}
$$

${ }^{12}$ See the website at https://www.worldometers.info/coronavirus/\#ref-23. 


$$
\tilde{\mu}_{T}^{\prime}=(\rho+m) \mu_{T}^{\prime}+(1-\eta) \frac{d^{2} a_{T}(T)}{d T^{2}} T^{\prime}(t, 0)
$$

where (') indicates the derivative with respect to $\varepsilon$ and $\mu_{T}$ is the costate variable associated with the temperature dynamics (24). Then,

$$
\left.J^{\prime}(\varepsilon)\right|_{\varepsilon=0}=\int_{0}^{\infty} \mathrm{e}^{-\rho t}\left[\eta \frac{E^{\prime}(t, 0)}{E^{2}}-(1-\eta)\left(\frac{d a_{T}(T)}{d T} T^{\prime}(t, 0)+\frac{d a_{I}(I)}{d I} a I^{\prime}(t, 0)\right)\right] d t .
$$

When $\frac{d a_{T}(T)}{d T}=a_{T}$ constant, then $\mu_{T}^{\prime}=0$, and thus $E^{\prime}=0$ and $T^{\prime}=0$. Then, with constant $f$, (29) implies that $I^{\prime}(t)=\mathrm{e}^{(\beta f-\gamma) t}$ and (33) is reduced to

$$
\left.J^{\prime}(\varepsilon)\right|_{\varepsilon=0}=-\int_{0}^{\infty} \mathrm{e}^{-\rho t}\left[(1-\eta) \mathrm{e}^{(\beta f-\gamma) t}\right] d t=\frac{-(1-\eta)(d a(I) / d I)}{(\rho+\gamma-\beta f)} .
$$

\section{References}

Afelt A, Frutos R, Devaux C (2018) Bats, coronaviruses, and deforestation: toward the emergence of novel infectious diseases? Front Microbiol. https://doi.org/10.3389/fmicb.2018.00702

Becker G (1965) A theory of the allocation of time. Econ J 75:493-517

Barnett M, Brock WA, Hansen LP (2020a) Pricing uncertainty induced by climate change. Rev Financ Stud 33:1025-1066

Barnett M, Buchak G, Yannelis C (2020b) Epidemic responses under uncertainty. NBER Working Papers 27289, National Bureau of Economic Research

Birrell P, Blake J, van Leeuwen E (2020) COVID-19: nowcast and forecast. https://www.mrc-bsu.cam. ac.uk/now-casting/

Brock WA, Hansen LP (2018) Wrestling with uncertainty in climate economic models. In: Chari VV, Litterman R (eds) Climate change economics: the role of uncertainty and risk. Wiley, Hoboken

Brock WA, Xepapadeas A (2017) Climate change policy under polar amplification. Eur Econ Rev 94:263-282

Cline WR (2020) Transient climate response to cumulative emissions (TCRE) as a reduced-form climate model. Working Paper 20-02 May, 2020. https://econintl.com/working-papers/

Daily G, Ehrlich P (1996) Global change and human susceptibility to disease. Annu Rev Energy Environ 21:125-144

Greenstone M, Nigum V (2020) Does social distancing matter? https://bfi.uchicago.edu/working-paper /2020-26/

Greenstone M, Reguant M, Ryan N, Dobermann T (2019) Energy and environment, evidence paper, draft. https://www.theigc.org/wp-content/uploads/2019/12/IGC-Energy-evidence-paper-December-2019_ web.pdf

Harvey F (2020) Jane Goodall: humanity is finished if it fails to adapt after Covid-19. The Guardian, 3 June 2020. https://www.theguardian.com/science/2020/jun/03/jane-goodall-humanity-is-finished-if-it-fails -to-adapt-after-covid-19

Henriques M (2020) Will Covid-19 have a lasting impact on the environment? BBC, 27 March 2020. https:// www.bbc.com/future/article/20200326-covid-19-the-impact-of-coronavirus-on-the-environment

Hethcote HW (1976) Qualitative analyses of communicable disease models. Math Biosci 28:335-356

Hethcote HW (2000) The mathematics of infectious diseases. SIAM Rev 42:599-653

International Energy Agency (2020) Global energy and $\mathrm{CO}_{2}$ emissions in 2020. https://www.iea.org/reports/ global-energy-review-2020/global-energy-and-co2-emissions-in-2020\#abstract

MacDougal AH, Friedlingstein P (2015) The origin and limits of the near proportionality between climate warming and cumulative $\mathrm{CO}_{2}$ emissions. J Clim 28:4217-4230

Matthews HD, Gillett NP, Stott PA, Zickfield K (2009) The proportionality of global warming to cumulative carbon emissions. Nature 459:829-833 
Muehlenbein MP (2013) Human-wildlife contact and emerging infectious diseases. In: Brondızio ES, Moran EF (eds) Human-environment interactions: current and future directions, Chapter 4. Springer Science, Dordrecht

Nabi G, Siddique R, Ali A, Khan S (2020) Preventing bat-born viral outbreaks in future using ecological interventions. Environ Res. https://doi.org/10.1016/j.envres.2020.109460

Polasky S, Wagener F, de Zeeuw A (2010) Optimal management with potential regime shifts. J Environ Econ Manag 62:229-240

Pollak RA, Wachter ML (1975) The relevance of the household production function and its implications for the allocation of time. J Polit Econ 83:255-278

Ramon P (2020) The Amazon could easily be the next source of Coronaviruses, Scientist warns. Science Alert, 15 May 2020. https://www.sciencealert.com/amazon-could-be-next-virus-hot-zone-scientist

Shaw J (2020) COVID-19 may be much more contagious than we thought. Harvard Magazine, 13 May 2020. https://www.harvardmagazine.com/2020/05/r-nought

Tornatore E, Buccellato SM, Vetro P (2005) Stability of a stochastic SIR system. Physica A 354:111-126

van der Ploeg F, de Zeeuw A (2019) Pricing carbon and adjusting capital to fend off climate catastrophes. Environ Resour Econ 72:29-50

World Bank (2020) WDI data. http://wdi.worldbank.org/table/3.8

Zimmer K (2019) Deforestation is leading to more infectious diseases in humans. National Geographic, 22 November 2019. https://www.nationalgeographic.com/science/2019/11/deforestation-leading-to-moreinfectious-diseases-in-humans/

Publisher's Note Springer Nature remains neutral with regard to jurisdictional claims in published maps and institutional affiliations. 